\title{
Enhanced Electrocatalytic Oxidation of Glucose on Nickel Oxides/Graphene Nanoparticles Modified Glassy Carbon Electrode
}

\author{
M. I. Awad ${ }^{1,2, *}$, Maha E. Al-Hazemi ${ }^{3}$,Zahrah T. Al-thagafi, ${ }^{4}$ \\ ${ }^{1}$ Chem. Department, Faculty of Applied Sciences, Umm Al-Qura University, Makkah, Saudi Arabia. \\ ${ }^{2}$ Chem. Department, Faculty of Science, Cairo University, Cairo, Egypt. \\ ${ }^{3}$ Chem. Department, Faculty of Applied Sciences, Jeddah University, Jeddah, Saudi Arabia \\ ${ }^{4}$ Chem. Department, Faculty of Sciences, Taif University, Taif, Saudi Arabia. \\ *E-mail: $\underline{\text { mawad70@yahoo.com }}$
}

Received: 16 September 2021 / Accepted: 29 October 2021 /Published: 6 December 2021

\begin{abstract}
Graphene (Gr) modified glassy carbon (GCE) electrode with sequentially electrodeposited nickel oxide nanoparticles $\left(\mathrm{NiO}_{\mathrm{x}}\right)$ were prepared as effective and stable electrode for electrochemical oxidation of glucose. The electrochemical behavior of the thus prepared electrode towards glucose was examined by voltammetric techniques. The $\mathrm{NiO}_{\mathrm{x}} / \mathrm{Gr} / \mathrm{GCE}$ electrode was prepared by casted $\mathrm{Gr}$ onto an electrochemically activated GCE then $\mathrm{NiO}_{x}$ was deposited either from nickel bath containing glucose as additive or not. The thus modified electrode was applied for the electrooxidation of glucose in alkaline medium. The effect of loading level of nickel of modified electrode were investigated as well. Furthermore, the $\mathrm{NiO}_{\mathrm{x}} / \mathrm{Gr} / \mathrm{GCE}$ electrode was morphologically characterized using scanning electron microscopy (SEM). The elemental composition is probed using energy-dispersive X-ray spectroscopy (EDX). The crystallographic orientation is examined using X-Ray diffraction (XRD) technique. The embedding of $\mathrm{NiO}_{\mathrm{x}}$ in the $\mathrm{Gr}$ enhanced their activity and stability for glucose oxidation reaction. Moreover, the best electrocatalytic activity and stability for glucose was obtained at $\mathrm{NiO}_{\mathrm{x}}(\mathrm{Glu}) / \mathrm{Gr} / \mathrm{GCE}$ fabricated from nickel bath containing glucose as compared with the unmodified GCE electrode and $\mathrm{NiO}_{\mathrm{x}} / \mathrm{Gr} / \mathrm{GCE}$ in nickel bath-glucose free. This enhancement is attributed to a synergistic effect between $\mathrm{NiO}_{\mathrm{x}}(\mathrm{Glu})$ and $\mathrm{Gr}$, where the $\mathrm{NiO}_{\mathrm{x}}$ improve the electrochemical behavior via the formation of $\mathrm{Ni}^{3+}$ $(\mathrm{NiOOH})$. The stability of the electrode is enhanced by using grapheme as a substrate for the nickel oxyhydroxide $(\mathrm{NiOOH})$.
\end{abstract}

Keywords: Nickel- Nanoparticles- Glucose- Electrocatalysis-Graphene.

\section{FULL TEXT}

(C) 2022 The Authors. Published by ESG (www.electrochemsci.org). This article is an open access article distributed under the terms and conditions of the Creative Commons Attribution license (http://creativecommons.org/licenses/by/4.0/). 